\title{
Asymptomatic malaria and associated factors among blood donors in Mwanza, Tanzania
}

DOMENICA MORONA ${ }^{1 *}$, BETRAND MSEMWA 1 , MARIA M. ZINGA ${ }^{1}$, MARTHA F. MUSHI ${ }^{2}$, MARIAM M. MIRAMBO ${ }^{2}$ and STEPHEN E. MSHANA ${ }^{2}$

'Department of Medical Parasitology and Entomology, Weill Bugando School of Medicine, Catholic University of Health and Allied Sciences, P.O. Box 1464, Mwanza, Tanzania

${ }^{2}$ Department of Microbiology and Immunology, Weill Bugando School of Medicine, Catholic University of Health and Allied Sciences, Mwanza, Tanzania

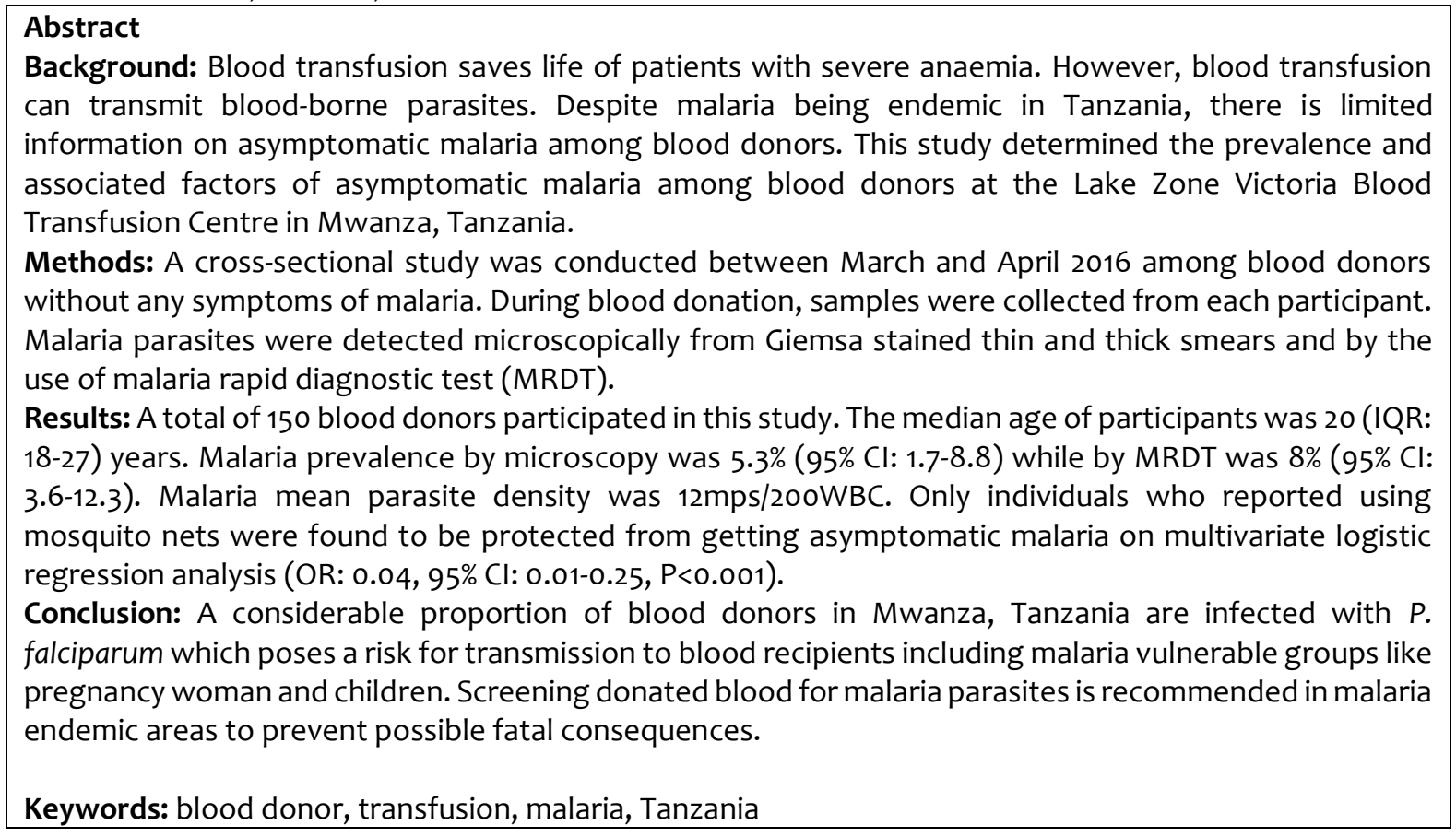

\section{Introduction}

Blood transfusion is a medical treatment used in the management of emergency cases such as injuries, surgeries and other life-threatening conditions. Despite being a lifesaving procedure, it may be a potential risk for transmitting blood-borne infections (Cheesbrough et al., 2006). Malaria parasites can be found in the blood of infected individuals and might be transmitted through exposure to an infected person's blood via blood transfusion, sharing needles or syringes contaminated with blood (Chamberland et al., 2001).

Malaria is responsible for millions of morbidity and thousands of deaths annually in developing countries. The most recent World Malaria Report indicates that there were 212 million new cases of malaria worldwide in 2015 (WHO, 2016), with the Sub-Saharan Africa accounting for $90 \%$ of the cases. During the same period of time, there was an estimated 429,000 malaria deaths worldwide (WHO, 2016). In Tanzania, malaria is the most important cause of morbidity and mortality. A recent population-based survey indicates a $9 \%$ prevalence of malaria among children with the highest reported from the Lake Victoria Regions of Geita and Kagera (TDH-MIS, 2016). All human malaria parasites (Plasmodium falciparum, P. vivax, P. ovale and P. malariae) can be transmitted through blood transfusion (Muerhoff et al., 2010). To ensure the safety of blood before transfusion, the World Health Organization (WHO) recommends the blood collected for transfusion to be screened for presence of Hepatitis B Virus (HBV), Hepatitis C Virus (HBV), Syphilis and Human Immunodeficiency Virus (HIV) (Epstein \& Holmberg, 2010). In addition, in malaria

\footnotetext{
*Correspondence Email: dmorona@gmail.com
} 
endemic areas, it is advised to screen for blood-borne parasites (BBP) such as malaria before transfusion. Despite malaria being endemic in Africa, most blood transfusion centres do not routinely screen for malaria parasites (Uneke et al., 2006). Moreover, donated blood has also been used for the management of pregnancy-related anaemia, as well as in children in need. Parallel to this, malaria has been documented to be one of the leading causes of adverse poor pregnancy outcomes and deaths among underfives in most of the resource-limited countries. This raises a concern, particularly in this era of campaigns to control malaria and associated adverse outcomes in a resource-limited set-up. Previous studies in developing countries reported a prevalence of $26.5 \%$ and $1.5 \%$ among male and female blood donors respectively (Agboola et al., 2010; Oladeinde et al., 2014; Olawumi et al., 2015; Wariso \& Oboro, 2015). At Bugando Medical Centre in Mwanza, Tanzania about 3,020 blood units are transfused to patients yearly. These units are not routinely screened for malaria parasites and are usually transfused to different patients, including vulnerable groups like pregnant women and children.

This study was designed to determine the prevalence of asymptomatic malaria among blood donors, information which may be useful in recommending possible interventions such as introducing a policy of screening donated blood for malaria parasites.

\section{Materials and Methods}

\section{Study area and design}

A cross-sectional study was conducted in Mwanza City in north-western Tanzania, from March to April 2016 among blood donors at the Lake Victoria Zone Transfusion Centre. The Lake Victoria Zone Transfusion Centre is located in Mwanza City and serves all regions around the Lake Zone (Mwanza, Geita, Simiyu, Shinyanga, Tabora, Mara) and other regions like Kigoma and Singida. On average, about 100 to 250 units of blood are collected per week.

\section{Study population and sampling technique}

The study included adult blood donors, aged above 18 years, with a haemoglobin level of $>12.5 \mathrm{mg} / \mathrm{dl}$ and a weight of $>50 \mathrm{~kg}$. All donors with a history of anti-malarials use in the past three months were excluded. The sample size was calculated using the formula by Kish (1965) and a prevalence of $10.2 \%$ was used. The minimum sample size obtained was 140 . A total of 150 blood donors were enrolled.

\section{Data collection and laboratory processes}

A structured data collection tool was used to collect socio-demographic and other relevant information. About $2 \mathrm{ml}$ of blood was collected in EDTA tube and transferred to CUHAS Multipurpose Laboratory for processing. Thick films were prepared for all blood specimens, air dried and stained with Giemsa stain (Sigma Aldrich, Nairobi Kenya). In addition, a thin film was prepared for all specimens, air-dried then fixed with methanol before staining with Giemsa stain. All smears were examined microscopically starting with a low power (10x objective) for field then high power (100x oil immersion objective) for the parasite detection and differentiation of intracellular parasites to species level. In addition, malaria Rapid Diagnostic Test (MRDT) $\left(\text { CareStart }{ }^{T M}\right)^{R}$ was used to detect the presence of malaria antigen in all specimens. In this study, the positive malaria test was defined by either having malaria parasitaemia or a positive malaria rapid test result.

\section{Data analysis}

All data were recorded in Excel sheet for coding and cleaning then transferred to STATA version 11 for analysis. Results were presented into proportions for categorical variables and median with interquartile range (IQR) for continuous variables. Wilcoxon (Man Whitney) rank sum test was 
used to compare median between the groups. Backward stepwise elimination logistic regression model was used to determine the predictors for asymptomatic malaria.

\section{Ethical considerations}

The protocol for conducting the study was approved by the Joint Bugando Medical Centre-Catholic University of Health and Allied Ethics and Review Committee with ethical clearance number CREC 136/2016. The protocol and importance of the study was explained to all participants and a written informed consent was obtained from each participant prior to their enrolment in the study.

\section{Results}

\section{Socio-demographic characteristics of the study participants}

A total of 150 adults aged 17 to 57 years were enrolled in the study between March and April 2016. The median age of study participants was 20 (IQR: 18-27) years. The majority of the participants $133(88.7 \%)$ were males, and individuals residing in urban areas formed $70.7 \%$ of the study population. Students, 98 (65.3\%), formed the majority of the study population (Table1).

Table 1: Socio-demographic characteristics of the study participants

\begin{tabular}{lll}
\hline Characteristics & Response & Frequency (\%)/ Median \\
\hline Age & Male & $133(88.7)$ \\
Residence & Female & $11(11.3)$ \\
& Rural & $44(29.3)$ \\
Occupation & Urban & $106(70.7)$ \\
& Students & $98(65.3)$ \\
& Others & $52(34.7)$ \\
\hline
\end{tabular}

*Continuous variable

\section{Prevalence and factors associated with asymptomatic malaria}

Malaria prevalence by microscopy was 5.3\% (95\% Cl: $1.7-8.8$ ) while by MRDT was $8 \%$ (95\% Cl: 3.6 12.3). All patients found positive by microscopy were also positive by MRDT. The geometric mean parasite density of 12/200WBC. On Wilcoxon (Man Whitney) rank sum test, there was no significant difference between the median age of positive cases and those of negative cases (19.5, IQR 18.522 years versus 20 , IQR: $18-28$ years, $\mathrm{P}=0.405$ ).

Table 2: Univariate and multivariate logistic regression analysis of the factors associated with asymptomatic malaria (using MRDT results)

\begin{tabular}{|c|c|c|c|c|c|c|}
\hline Characteristic & Response & Positivity (\%) & $\begin{array}{l}\text { Univariate OR } \\
(95 \% \mathrm{Cl})\end{array}$ & P-value & $\begin{array}{l}\text { Multivariate } \\
\text { OR }(95 \% \mathrm{Cl})\end{array}$ & P-value \\
\hline Age* & & $19.5(\mathrm{IQR}=18.5-22)$ & $0.88(0.75-1.03)$ & 0.133 & $0.83(0.68-1.01)$ & 0.063 \\
\hline \multirow[t]{2}{*}{ Sex } & Female (17) & $1(5.88)$ & 1 & & & \\
\hline & Male (133) & $11(8.27)$ & $0.69(0.08-5.73)$ & & & \\
\hline \multirow[t]{2}{*}{ Residence } & Rural (44) & $3(6.82)$ & 1 & & & \\
\hline & Urban (106 & $9(8.49)$ & $1.27(0.32-4.92)$ & 0.734 & & \\
\hline \multirow[t]{2}{*}{ Occupation } & Others (52) & $3(5.77)$ & 1 & & & \\
\hline & Student (98) & $9(9.18)$ & $1.65(0.42-6.39)$ & 0.467 & & \\
\hline \multirow[t]{2}{*}{ Mosquito bite } & No (4) & $0(0.00)$ & 1 & & & \\
\hline & Yes (146) & $12(8.22)$ & - & & & \\
\hline \multirow[t]{2}{*}{ Mosquito net use } & No (9) & $4(44.44)$ & 1 & & & \\
\hline & Yes (141) & $8(5.67)$ & $0.08(0.01-0.33)$ & 0.001 & $0.04(0.01-0.25)$ & 0.001 \\
\hline
\end{tabular}

*Continuous variable; insect bite was found to be perfect predictor

On univariate logistic regression analysis, individuals who were using mosquito nets were significantly protected from getting malaria (OR: $0.08,95 \% \mathrm{Cl}: 0.01-0.33, \mathrm{P}<0.001$ ). As the age increases, the prevalence of malaria was found to decrease (OR: $0.88,95 \% \mathrm{Cl}: 0.75-1.03, \mathrm{P}=0.133$ ). 
Only individuals who were using mosquito nets were found to be protected from getting malaria when adjusted to age (OR: $0.04,95 \% \mathrm{Cl}: 0.01-0.25, \mathrm{P}<0.001$ ) (Table 2).

\section{Discussion}

This is the first study to report the prevalence of asymptomatic malaria among blood donors in Tanzania. The majority of the participants recruited in this study were males, most residing in urban areas. The current study population is similar to that of previous studies in Nigeria (Oladeinde et al., 2014; Olawuni et al., 2015; Wariso \& Oboro, 2015) but dissimilar to that of other studies where females predominated (Owusu-Ofori et al., 2010; Muntaka \& Opoku-Okrah, 2013). This could be explained by the fact that in this setting blood is mostly donated to save vulnerable groups such as pregnant women and children and, traditionally, males are more involved than females. Regarding occupation, the majority of the participants in the current study were students. This is in agreement with a previous report in Nakuru, Kenya, which had a similar observation (Bartonjo, 2013). The predominance of students and youths is due to the fact that, in Tanzania, most of the blood donation campaigns target youths or students who are easier to mobilise and perceived to be a healthy young generation which can save other groups.

In the current study, the prevalence of asymptomatic malaria was found to be $8 \%$. This is comparable to other studies in Ghana which showed a range of prevalence between $10 \%$ and $13.7 \%$ (Okocha et al., 2005; Owusu-Ofori et al., 2016). However, the reported prevalence in this study is lower compared to previous reports in Kenya, Ethiopia and Nigeria (Okocha et al., 2005; Erhabor et al., 2007; Epidi et al., 2008; Bartonjo, 2013; Olawuni et al., 2015). Though, in comparison to previous studies in the general population, the prevalence reported in this study is high (van Eijk et al., 2003; Nzobo et al., 2015). This could be explained by geographical variations in malaria transmission.

The parasitaemia indicates a potential risk for malaria transmission to vulnerable groups in this setting. Considering the fact that the majority of the blood recipients are groups vulnerable to malaria such as pregnant women and children (Epidi et al., 2008; Owusu et al., 2010), screening for malaria during blood donation should be taken into consideration to avoid possible consequences.

In our study, all positive cases were due to $P$. falciparum infection. This could be explained by the fact that $P$. falciparum is the predominant species in Tanzania (Mboera, 2000). Considering the current efforts to control malaria in the tropics and the fact that routinely available diagnostic techniques such as microscopy and rapid tests can be used, there is a paramount need to consider screening for malaria parasites in all donated blood. The findings showed that as the age increases, the prevalence of asymptomatic malaria was found to decrease with borderline significance. This could be explained by that fact that in endemic areas like Tanzania, the majority of individuals, particularly under-fives, are infected repeatedly hence mounting a partial immunity to malaria parasites that prevents them from getting numerous episodes of malaria in adulthood (Menendez, 2006; Takem \& D’Alessandro, 2013).

The importance of using mosquito nets is emphasized by the data of the current study whereby individuals who were using mosquito nets were found to be protected from getting malaria on multivariate logistic regression analysis. This emphasizes the need to continue with various malaria control interventions across the country including the promotion of the use of treated mosquito nets as previously documented (Bhattarai et al., 2007; Killeen et al., 2007).

A considerable proportion of blood donors in Mwanza were infected with P. falciparum which poses a risk for transmission to blood recipients including malaria vulnerable groups like pregnancy woman and children. Screening donated blood for malaria parasites is recommended in malaria endemic areas to prevent possible fatal consequences. Treatment with riboflavin and /or UV irradiation to inactivate malaria parasites can be alternative strategies. Further studies on other blood-borne parasites in this population are highly recommended. 


\section{Competing interest}

None declared

\section{Author's contributions}

MMM, MFM, SEM and DM participated in the design of the work. BM did data collection and laboratory work. SEM, MFM and MMM performed statistical analysis and interpretation of the data. MMM wrote the first draft of the manuscript. SEM and DM provided critical revision of the manuscript. All authors read and approved the final version of the manuscript.

\section{Acknowledgements}

The authors would like to acknowledge the technical support provided by Mr. Emmanuel Mkumbo of Catholic University of Health and Allied Sciences for his technical assistance in carrying the malaria rapid diagnostic tests.

\section{References}

Agboola, T., Ajayi, M., Adeleke, M. \& Gyang, P. (2010) Prevalence of malaria parasite among blood donors in Lagos University Teaching Hospital, Lagos Nigeria. Annals of Biological Research 1:72-75.

Bartonjo, G. (2013) Prevalence and factors associated with transfusion transmissible infections among blood donors at Regional Blood Transfusion Center, Nakuru and Tenwek Mission Hospital, Kenya. MSc Thesis, Jomo Kenyatta University of Agriculture and Technology. http://ir.jkuat.ac.ke/bitstream/handle/123456789/1902/

Bhattarai, A., Ali, A.S., Kachur, S.P., Mårtensson, A., Abbas, A.K., Khatib, R., Al-Mafazy, A-W., Ramsan, M., Rotllant, G., Gerstenmaier, J.F. (2007) Impact of artemisinin-based combination therapy and insecticide-treated nets on malaria burden in Zanzibar. PLoS Medicine 4(11):e309.

Cheesbrough, M. (2006) District Laboratory Practice in Tropical Countries. Cambridge University Press.

Chamberland, M.E., Alter, H.J., Busch, M.P., Nemo, G. \& Ricketts, M. (2001) Emerging infectious disease issues in blood safety. Emerging Infectious Diseases 7(3 Suppl):552.

Epidi, T., Nwani, C. \& Ugorji, N. (2008) Prevalence of malaria in blood donors in Abakaliki Metropolis, Nigeria. Scientific Research and Essays 2008, 3(4):162-164.

Epstein, J.S. \& Holmberg, J.A. (2010) Progress in monitoring blood safety. Transfusion 50: 1408-1412.

Erhabor, O., Ok, O., Awah, I., Uko, K.E., Charles, A.T. (2007) The prevalence of Plasmodia parasitaemia among donors in the Niger delta of Nigeria. Tropical Doctor 37: 32-34.

Killeen, G.F., Smith, T.A., Ferguson, H.M., Mshinda, H., Abdulla, S., Lengeler, C., Kachur, S.P. (2007) Preventing childhood malaria in Africa by protecting adults from mosquitoes with insecticide-treated nets. PLoS Medicine 4(7):e229.

Kish, L. (1965) Survey Sampling. New York: John Wiley and Sons, Inc.

Mboera, L.E.G. (2000) Fifty Years of Health Research in Tanzania (1949-1999). Annotated Bibliography. DUP (1996) Ltd., 373pp.

Menendez, C. (2006) Malaria during pregnancy. Current Molecular Medicine 6(2):269-273.

Muerhoff, A.S., Birkenmeyer, L.G., Coffey, R., Dille, B.J., Barnwell, J.W., Collins, W.E., Sullivan, J.S., Dawson, G.J. \& Desai, S.M. (2010) Detection of Plasmodium falciparum, P. vivax, P. ovale, and $P$. malariae merozoite surface protein 1-p19 antibodies in human malaria patients and experimentally infected nonhuman primates. Clinical and Vaccine Immunology 17: 1631-1638. 
Muntaka, S. \& Opoku-Okrah, C. (2013) The prevalence of malaria parasitaemia and predisposition of $\mathrm{ABO}$ blood groups to Plasmodium falciparum malaria among blood donors at a Ghanaian Hospital. AU Journal of Technology 16(4).

Nzobo, B.J., Ngasala, B.E. \& Kihamia, C.M. (2015) Prevalence of asymptomatic malaria infection and use of different malaria control measures among primary school children in Morogoro Municipality, Tanzania. Malaria Journal 14(1):1.

Okocha, E., Ibeh, C., Ele, P. \& Ibeh, N. (2005) The prevalence of malaria parasitaemia in blood donors in a Nigerian teaching hospital. Journal of Vector Borne Diseases 42: 21.

Oladeinde, B.H., Omoregie, R., Osakue, E.O. \& Onaiwu, T.O. (2014) Asymptomatic malaria among blood donors in benin city Nigeria. Iranian Journal of Parasitology 9: 415.

Olawumi, H.O., Fadeyi, A., Babatunde, S.K., Akanbi, I.I., Babatunde, A.S., Sani, M.A. \& Aderibigbe, S.A. (2015) Malaria parasitaemia among blood donors in llorin, Nigeria. African Journal of Infectious Diseases 9: 10-13.

Owusu-Ofori, A., Gadzo, D. \& Bates, I. (2016) Transfusion-transmitted malaria: donor prevalence of parasitaemia and a survey of healthcare workers knowledge and practices in a district hospital in Ghana. Malaria Journal 15(1):1.

Owusu-Ofori, A.K., Parry, C. \& Bates, I. (2010) Transfusion-transmitted malaria in countries where malaria is endemic: a review of the literature from sub-Saharan Africa. Clinical Infectious Diseases 51: 1192-1198.

Takem, E.N. \& D'Alessandro, U. (2013) Malaria in pregnancy. Mediterranean Journal of Hematology and Infectious Diseases 5: 2013010.

TDH-MIS (2016) Tanzania Demographic and Health Survey and Malaria Indicator Survey. Ministry of Helath, Community Development, Gender, Elderly and Children (Tanzania Mainland), Ministry of Health (Zanzibar), National Bureau of Statistics, Office of the Chief Government Statistician, and ICF. Dar es Salaam, Tanzania and Rockville, Maryland, USA.

Uneke, C.J., Ogbu, O. \& Nwojiji, V. (2006) Potential risk of induced malaria by blood transfusion in South-eastern Nigeria. McGill Journal of Medicine 9(1):8.

van Eijk, A.M., Hill, J., Noor, A.M., Snow, R.W. \& ter Kuile, F.O. (2015) Prevalence of malaria infection in pregnant women compared with children for tracking malaria transmission in subSaharan Africa: a systematic review and meta-analysis. Lancet Global Health 3(10):e617e628.

Wariso, K.T. \& Oboro, I.L. (2015) Diagnosis of malaria among blood donors in Port Harcourt, Nigeria: microscopy or rapid diagnostic tests?Advances in Microbiology 5(05):358.

WHO (2016) World Malaria Report 2016. http://www.who.int/malaria/media/world-malaria-report2016/en/ 